\title{
Erratum: Pathogen subversion of cell-intrinsic innate immunity
}

Craig R Roy \& Edward S Mocarski

Nat. Immunol. 8, 1179-1187 (2007); published online 19 October 2007; corrected after print 17 December 2007

In the version of this article initially published, some of the reference numbering in the list and text is incorrect, and a reference is missing. The errors have been corrected in the HTML and PDF versions of the article.

\section{Corrigendum: The MAPK-activated kinase Rsk controls an acute Toll-like receptor signaling response in dendritic cells and is activated through two distinct pathways

\begin{abstract}
Rossana Zaru, Natalia Ronkina, Matthias Gaestel, J Simon C Arthur \& Colin Watts
Nat. Immunol. 8, 1227-1235 (2007); published online 30 September 2007; corrected after print 16 November 2007

In the version of this article initially published, the Author Contributions section was incorrect. The correct author contributions are as follows: R.Z and C.W designed the study, interpreted the data and wrote the manuscript; R.Z. did the experiments; N.R. and M.G. provided MK2-null mice and generated embryonic stem cells carrying a targeted allele of MK3; J.S.C.A. provided advice and MSK1-MSK2-null mice and generated MK3-null mice from the MK3 embryonic stem cells provided by N.R. and M.G. The error has been corrected in the PDF version of the article.
\end{abstract}

\section{Corrigendum: Comprehensive epigenetic profiling identifies multiple distal regulatory elements directing transcription of the gene encoding interferon- $\gamma$}

Jamie R Schoenborn, Michael O Dorschner, Masayuki Sekimata, Deanna M Santer, Maria Shnyreva, David R Fitzpatrick, John A Stamatoyannopoulos \& Christopher B Wilson

Nat. Immunol. 8, 732-742 (2007); published online 3 June 2007; corrected after print 16 November 2007

In the version of this article initially published, labels in Figures 2 and 4 are incorrect (as is the relevant text for Figure 4), and a reagent is incorrectly identified in Methods. In Figure 2, the label "IfngCNS+54" should be "IfngCNS+55." For Figure 4, the primers used to analyze the intronic region of Ifng for CpG methylation after bisulfite treatment amplify intron 1; thus, the label above the sixth column in Figure 4a should be "Ifng intron 1" and the accompanying text on page 736, column 1, should read "intron 1" on lines 3 and 13 . In the Methods section, page 740 column 2 , the end of line 23 should read "rabbit immunoglobulin G." The errors have been corrected in the PDF version of the article.

\section{Corrigendum: Suppression of autoimmune inflammation of the central ner- vous system by interleukin 10 secreted by interleukin 27-stimulated T cells}

Denise C Fitzgerald, Guang-Xian Zhang, Mohamed El-Behi, Zoë Fonseca-Kelly, Hongmei Li, Shuo Yu, Christiaan J Saris, Bruno Gran, Bogoljub Ciric \& Abdolmohamad Rostami

Nat. Immunol. 8, 1372-1379 (2007); published online 11 November 2007; corrected after print 28 November 2007

In the version of this article initially published, the initials for the seventh author are incorrect. The correct author name is Christiaan J M Saris. The error has been corrected in the HTML and PDF versions of the article. 